\title{
CURSOS BREVES EN EL EXTRANJERO: APRENDIZAJES LOGRADOS POR ESTUDIANTES DE POSGRADO EN EDUCACIÓN
}

\author{
CURSOS DE CURTA DURAÇÃO NO EXTERIOR: APRENDIZAGENS OBTIDAS POR \\ ESTUDANTES DE PÓS-GRADUAÇÃO EM EDUCAÇÃO
}

\author{
SHORT COURSES ABROAD: LEARNINGS OBTAINED BY POSTGRADUATE \\ STUDENTS IN EDUCATION
}

\author{
José Luis BONILLA ESQUIVEL ${ }^{1}$ \\ Melanie Elizabeth MONTES SILVA ${ }^{2}$
}

RESUMEN: La movilidad académica es una estrategia para lograr la internacionalización y contribuye a responder a las exigencias de la globalización. Este trabajo reporta los aprendizajes logrados por 31 estudiantes mexicanos de un programa profesionalizante de Maestría en Educación, quienes tomaron un curso breve, de una semana, en la Universidad de Salamanca y en la Universidad de Coimbra. El método consistió en realizar análisis de contenido cualitativo a los trabajos finales de los estudiantes, específicamente a los apartados reflexivos que les fueron solicitados. El resultado permite identificar aprendizajes de naturaleza diversa: (1) conceptuales, principalmente los propios del curso; (2) procedimentales, por la planeación del viaje; (3) actitudinales, como crecimiento personal, vivencia de valores y reproducción de estereotipos, y (4) socioafectivos, por la convivencia. Como conclusión se establece la relevancia y significado de viajes de estudio de este tipo, que, a pesar de su brevedad, conllevan aprendizajes cuantiosos y de diversa naturaleza.

PALABRAS CLAVE: Internacionalización. Movilidad estudiantil. Estudiante de Posgrado. Aprendizaje.

RESUMO: A mobilidade acadêmica é uma estratégia para alcançar a internacionalização e ajuda a atender às demandas da globalização. Este trabalho relata os aprendizados alcançados por 31 estudantes mexicanos de um programa de mestrado profissionalizante, que fez um curso curto de uma semana na Universidade de Salamanca e na Universidade de Coimbra. O método consistiu na realização da análise de conteúdo qualitativo ao trabalho final dos alunos, especificamente as seções reflexivas solicitadas. O resultado permite identificar aprendizados de natureza diferente: (1) conceitual, principalmente os do curso; (2) processual, pelo planejamento da viagem; (3) atitudes, como crescimento pessoal, experiência de valores e reprodução de estereótipos, e (4) sociofetiva, pela convivência. A conclusão estabelece a relevância e a significância das viagens de estudo desse tipo, que, apesar de sua brevidade, envolvem grandes aprendizados de natureza diferente.

${ }^{1}$ Centro de Enseñanza Técnica y Superior (CETYS), Tijuana - Baja California - México. Director del Colegio de Ciencias Sociales y Humanidades y coordinador académico del Doctorado en Educación a nivel sistema. ORCID: https://orcid.org/0000-0003-1944-857X3. E-mail: joseluis.bonilla@cetys.mx

${ }^{2}$ Centro de Enseñanza Técnica y Superior (CETYS), Tijuana - Baja California - México. Coordinadora académica del Doctorado en Educación. ORCID: https://orcid.org/0000-0002-0499-0537. E-mail: melanie.montes@cetys.mx

RIAEE - Revista Ibero-Americana de Estudos em Educação, Araraquara, v. 15, n. esp. 4, p. 2577-2586, dez., 2020. e-ISSN: 1982-5587 
PALAVRAS-CHAVE: Internacionalização. Mobilidade estudantil. Estudante de pósgraduação. Aprendizagem.

ABSTRACT: Academic mobility is a strategy for achieving internationalization and helps to meet the demands of globalization. This paper reports the learning achieved by 31 Mexican students from a professional Master's program in Education, who took a short, one-week course at the University of Salamanca and the University of Coimbra. The method consisted in performing qualitative content analysis of the students' final papers, specifically the reflective sections that were requested. The result allows identifying learning of diverse nature: (1) conceptual, mainly those of the course; (2) procedural, by the planning of the trip; (3) attitudinal, as personal growth, experience of values and reproduction of stereotypes, and (4) socio-affective, by the coexistence. As a conclusion, the relevance and meaning of study trips of this type is established, which, in spite of their brevity, entails substantial learning of diverse nature.

KEYWORDS: Internationalization. Student mobility. Postgraduate student. Learning.

\section{Internacionalización y movilidad estudiantil}

Las instituciones de Educación Superior no se pueden limitar al ámbito de lo local, por ello se espera que adopten dimensiones internacionales e interculturales para alcanzar el diálogo e interacción de la enseñanza, la investigación y el servicio (LANDINELLI, 2010). En este contexto se vuelve relevante hablar de la internacionalización y de la movilidad académica.

De acuerdo con Knight (2015), el término internacionalización no es nuevo, pero sí debe ser claramente entendido y reformulado para atender diversas dimensiones que caracterizan el siglo XXI. Para tal efecto, la autora propone la siguiente definición de trabajo para este concepto: "La internacionalización a nivel nacional, sectorial e institucional se define como el proceso de integración de una dimensión internacional, intercultural o global en el propósito, las funciones o la entrega de la educación postsecundaria" (KNIGHT, 2015, p. 2). La internacionalización es, además, una forma de responder a las exigencias de la globalización, por lo que las instituciones de educación superior la establecen como parte de sus planes de desarrollo (ALCÓN, 2011), aunque cabe precisar que la internacionalización no debe ser considerada una meta en sí misma (DE WIT, 2011), sino que debe servir para que los estudiantes desarrollen competencias sobre aspectos globales, internacionales e interculturales.

Para que cumpla su propósito, la internacionalización puede ser concretada de diferentes formas en las instituciones educativas. Por ejemplo, se puede hablar de la internacionalización en el hogar, que consiste en integrar, de manera intencional, lo internacional y lo intercultural al plan de estudios, para que participen todos los estudiantes en entornos domésticos de 
aprendizaje (BEELEN; JONES, 2015). Asimismo, existe la internacionalización del currículo, en la que se integran las dimensiones de lo intercultural, internacional y global a los resultados del currículo, esto es, a los resultados de aprendizaje, los métodos de enseñanza, la evaluación y los servicios de apoyo, con el propósito de que los estudiantes desarrollen habilidades sociales y profesionales que les permitan desenvolverse en contextos internacionales y multiculturales (GREEN; MERTOVA, 2016; LEASK, 2015). Otra forma de promover la internacionalización es la más ampliamente difundida, la movilidad, ya sea de estudiantes, académicos, investigadores, directivos o personal administrativo (CHANTRAIN, 2010; FRANCO-LEAL; SOETANTO; CAMELO-ORDAZ, 2016; GARCÍA, 2013). Específicamente en la movilidad estudiantil,

\begin{abstract}
los estudiantes de licenciatura y posgrado realizan prácticas, cursos cortos y residencias académicas fuera de su institución. Si la estancia se cumple en un país extranjero constituye un instrumento importante para la formación integral del futuro profesional, la oportunidad de que aprenda otro idioma, conozca y conviva con personas pertenecientes a culturas diferentes.

Igualmente, permite aprovechar la presencia de estudiantes extranjeros $-\mathrm{o}$ de los nacionales que regresan del extranjero con diversas experiencias- para enriquecer a los educandos locales (ANUIES, [21--]).
\end{abstract}

En este trabajo se presenta la experiencia de movilidad estudiantil que vivió un grupo de 31 estudiantes inscritos en un programa de Maestría en Educación, ofrecido en CETYS Universidad. El objetivo que se busca alcanzar es identificar los aprendizajes que adquirieron los estudiantes con su experiencia de internacionalización.

\title{
Internacionalización en CETYS Universidad: curso de verano de la Maestría en Educación
}

CETYS Universidad es una institución privada que cuenta con tres campus en el Noroeste de México. Ofrece programas en diferentes áreas de conocimiento a nivel preparatoria, licenciatura, posgrado y educación continua. Para la institución, la internacionalización es uno de los elementos diferenciadores y, como tal, se ofrece a los estudiantes de todos los niveles educativos. La meta con este elemento es lograr la “internacionalización para todos” (GÁRATE; ROCHA, 2011, p. 42) mediante diversas estrategias.

En el caso de la Maestría en Educación, que es un programa profesionalizante, una de las formas en las que se logra la internacionalización es mediante la movilidad estudiantil, específicamente con estancias cortas en el extranjero, las cuales se ofrecen anualmente. Aunque 
las estancias cortas se promueven entre todos los estudiantes inscritos al programa, participan quienes están en condiciones y deciden hacerlo. Esto implica realizar el pago por la matrícula y, adicionalmente, absorber los gastos propios del viaje. Al curso de verano de 2017 asistieron 31 estudiantes. El papel de la institución ante los estudiantes es coordinar las actividades académicas, así como algunas actividades sociales y culturales complementarias, las cuales son responsabilidad de u profesor asignado como acompañante. El docente responsable da información sobre el curso y el viaje, da seguimiento al proceso de inscripción y viaja con los estudiantes para fungir como guía, enlace y asesor.

La temática de la asignatura cambia anualmente, según las opciones de las instituciones receptoras, pero siempre se hace equivalente por una materia del programa, pues la cantidad de horas es similar y el tema se vincula con los contenidos del programa. En 2017 se ofreció el curso Neurociencia en la educación, con duración de cinco sesiones organizadas en una semana. Generalmente el curso se hace en una universidad de España, pero gracias a los convenios y negociaciones institucionales, por primera vez fueron dos las instituciones receptoras: la Universidad de Salamanca, en España, en donde el grupo participó en actividades durante tres días, y la Universidad de Coimbra, en Portugal, donde los estudiantes realizaron actividades por dos días.

La dinámica se planeó de forma tal que los profesores expertos presentaran sesiones magistrales sobre los diversos aspectos de la temática propuesta. La asistencia a clases y el cumplimiento con lo solicitado en ellas representaba el 60\% de la calificación del curso; el 40\% restante se determinó con la calificación asignada a un trabajo final integrador, en el que se solicitaron los siguientes elementos: (1) reporte de los temas abordados en cada una de las sesiones; (2) recuperación, reflexión e identificación de los principales aprendizajes adquiridos, y (3) un apartado opcional, a manera de bono, en el que podían compartir cuestiones adicionales, por ejemplo, sus experiencias y los retos que enfrentaron.

\section{Método}

A fin de conocer la perspectiva de los participantes sobre los aprendizajes que lograron con el curso internacional de verano, se analizaron los trabajos que generaron como reporte final. Específicamente en las dos secciones que reflexionaban sobre sus aprendizajes.

Una vez conformado el corpus por los textos de los 30 estudiantes que entregaron el trabajo final, se procedió a realizar un análisis de contenido cualitativo (MAYRING, 2014). En el primer ciclo de codificación se recurrió a un análisis de tipo deductivo, el segundo fue de 
tipo inductivo (Saldaña, 2016). Esto es, en un primer momento se buscó categorizar los aprendizajes según su tipo de contenido: (1) conceptuales, que son hechos o conceptos; (2) procedimentales, vinculados con procedimientos o técnicas; (3) actitudinales, que son actitudes, valores o normas, y (4) contenidos socioafectivos, vinculados con las habilidades sociales, afectivas y comportamentales (GALLEGO; SALVADOR, 2009, p. 144-145). Posteriormente los datos fueron nuevamente categorizados, en un segundo ciclo de codificación; esta vez, con base en la información que emergió en los trabajos de los estudiantes.

La presentación de los resultados se hará de forma descriptiva, señalando los principales aspectos derivados del análisis, y se enriquecerá con la recuperación de fragmentos extraídos textualmente de los trabajos elaborados por los estudiantes.

\section{Resultados}

Los trabajos analizados reflejan que los estudiantes de la Maestría en Educación que participaron en el curso de verano en el extranjero tienen una valoración positiva de la experiencia. La describen como "gratificante", señalan que despertó su "asombro" y manifiestan sentirse "satisfechos". En cuanto a la institución, reconocen que contaron con acompañamiento durante el proceso, desde "la primera sesión informativa hasta que finalizó el viaje”; también identifican que se les hizo “"presión” para el envío de todos los requisitos” y agradecen que el docente acompañante les diera consejos de viaje, interactuara en un ambiente de "confianza para expresar dudas" y promoviera una "excelente comunicación". En cuanto a su experiencia personal, algunos valoran que por primera vez viajaron a Europa y hay quienes se manifestaron impresionados, por ejemplo, porque "por los pasillos de la Universidad de Salamanca se pasearon Miguel de Cervantes Saavedra y Cristóbal Colón”, por la infraestructura de los laboratorios y por tener "la oportunidad de conocer una de las bibliotecas más impresionantes en el mundo".

Hay estudiantes que señalan apreciar la calidad y calidez del trabajo de los docentes que los recibieron, como se muestra en el siguiente fragmento: "[un] aspecto que aprendí fue la humildad de los maestros, la disposición para dar tiempo de calidad, no solamente en el curso, sino al invitar a sus alumnos a comer". En cambio, otros estudiantes comparan y aprecian lo que se ofrece en su propia universidad, como se evidencia en lo expuesto por un estudiante: "me atrevo a decir que varios de nuestros maestros superan en calidad y contenido a lo que pudimos vivir en el extranjero". 
Además de las experiencias, un aspecto relevante es que todos los estudiantes consideran que lograron aprendizajes. Enseguida se clasifican los aprendizajes identificados, organizados según el tipo de contenido al que corresponden.

\section{Aprendizajes de contenidos conceptuales}

Los aprendizajes de tipo conceptual son los que más destacan, pues los estudiantes hacen una recuperación de los temas aprendidos, como neurociencias, neuroeducación, dislexia, aprendizaje, entre otros. Además, hacen alusión a frases textuales que se mencionaron en el curso y fueron significativas para ellos, como estas: "No solo se necesitan profesores que enseñen, también hay que lograr que los alumnos aprendan" o "Las neurociencias nos dicen que hay tantas formas de aprender como personas hay en el mundo".

Algo que destaca es que, mientras algunos aspectos del curso fueron significativos, relevantes y atractivos para algunos participantes, para otros resultaron confusos o poco atractivos, por ejemplo, el tema del "cientificismo" y la relación entre neurociencias y educación. Esta percepción diferenciada de los contenidos del curso se debe a tres factores principales. En primer lugar, a la formación previa de los estudiantes. A quienes no tenían conocimientos previos sobre los temas el curso les resultó particularmente complicado; en cambio, quienes sí tenían conocimientos previos asimilaron los temas de mejor manera, como ocurrió con un estudiante de la Maestría en Educación que es médico y pudo "comprender mejor la información que abordaban los científicos". En segundo lugar, un factor importante fueron sus intereses personales o el gusto que cada quien tiene ante ese tipo de temas, como ocurrió con una estudiante quien señala: "En lo personal no soy fan de la ciencia y debo mencionar que sí se me dificultó un poco entenderla". En tercer lugar, se puede señalar como factor a considerar su motivación para participar en la estancia, pues hay quienes reconocen interés por la temática, mientras que otros señalan otras motivaciones, como evidencia lo reportado por una estudiante: "decidí tomar el curso de verano, en primera instancia y siendo honesta, para evitar tomar una materia y tener fines de semana libres".

También es relevante que algunos identificaron posibles formas de transferir sus aprendizajes, sobre todo quienes son docentes, mientras que hay quien ve una aplicación más amplia, pues considera que

los docentes, las instituciones y autoridades correspondientes deben tener consciencia de la importancia de los procesos neuronales, no solo para poder trasmitir conocimiento dentro del aula, sino también para 
el manejo administrativo de las mismas entidades escolares, las relaciones con otros profesores y con la sociedad misma.

Por supuesto que también hubo aprendizajes conceptuales de aspectos no académicos, pero sí culturales. Por ejemplo, un estudiante aprendió que “a las cervezas se les llaman 'cañas', a los popotes 'paja', los bancos son ‘totas' y que las glorietas son 'rotondas””.

\section{Aprendizajes de contenidos procedimentales}

No se detectaron aprendizajes de procedimientos y técnicas vinculados con cuestiones académicas, lo cual es entendible porque las clases fueron eminentemente teóricas. No obstante, sí hubo aprendizajes procedimentales vinculados con la planeación y el desarrollo del viaje: logística de los lugares a visitar antes o después del curso, ahorrar por un lapso de hasta siete meses para poder costear el viaje, organizar la documentación necesaria, cumplir con los requisitos en los tiempos establecidos, organizar cuestiones laborales y familiares para poder realizar el viaje, usar el transporte público de otro país.

Además de las cuestiones ordinarias que implica un viaje internacional, se dio un caso extraordinario, como se aprecia en la siguiente descripción:

Definitivamente, este viaje y este curso dejaron importantes aprendizajes en mí. En la primer semana (sic.) en España fueron robados mi documentación y mi dinero. Al inicio me atemoricé y quise regresar de inmediato a México; sin embargo, hubiera sido muy cobarde si le hubiera sacado la vuelta al problema y no lo hubiera enfrentado. Fue dificil, ya que estaba solo [...], pero considero que mi cerebro desarrolló nuevas estrategias, o bien, a partir de la necesidad, mi cerebro creó nuevas conexiones para poder vivir ese tiempo.

Del fragmento anterior destacan dos cuestiones. Por un lado, hay una identificación explícita de aprendizajes adquiridos luego de la experiencia, los cuales pueden ser clasificados como de tipo procedimental. Por otro lado, se aprecia un esfuerzo intencionado de parte del estudiante por usar los conceptos aprendidos en el curso, y no fue el único caso, ocurre lo mismo con otros participantes del curso, aunque en contextos menos desafiantes.

\section{Aprendizajes de contenidos actitudinales}

Hay aprendizajes de índole personal, sobre todo vinculadas con la oportunidad de crecimiento, autodescubrimiento y empoderamiento, como se ilustra en el siguiente fragmento: 
Tuve que ser más organizada, aprender a confiar más en mí y en las decisiones que tomo, así mismo, el estar lejos de mi casa me ayudó a ser más independiente, saber ser ahorrativa, cuidarme y darme cuenta de que si puedo hacer las cosas sola, que no necesito estar acompañada de nadie para arriesgarme.

También se identifica la vivencia de valores, sobre todo en la interacción con los compañeros. Esto se evidencia en los cuando los estudiantes señalaron en sus trabajos que requirieron ser respetuosos, generosos, tolerantes y pacientes, especialmente con sus compañeros.

Asimismo, los estudiantes reforzaron actitudes y reprodujeron estereotipos con respecto a los habitantes de las ciudades que visitaron. Este tipo de comentarios fueron reiterativos, por ejemplo, en dos trabajos diferentes se presentaron las siguientes frases: "comprobé que no es un mito que los franceses huelen feo o que no se bañan" y "Sin duda el choque cultural fue más notorio con los españoles y su eterna baja autoestima". En el mismo trabajo del que se extrae el último fragmento se presentan una serie de frases que el estudiante afirma haber recibido durante su viaje por España, como esta: “Pero tú no estás negra, ¿segura que eres mexicana?”. Como se puede ver, los estereotipos se reproducen en ambos sentidos, según se vio en los trabajos generados por los estudiantes.

\section{Aprendizajes de contenidos socioafectivos}

Los aprendizajes de tipo socioafectivo son, después de los aprendizajes conceptuales, los que más identifican los estudiantes. Señalan haber aprendido a convivir con personas diferentes, con las que no hubieran pensado relacionarse, y lo hicieron tanto desde la planeación, como durante el viaje. Lo anterior se ilustra en el siguiente comentario:

conocer nuevos amigos-compañeros de aventura, con toda clase de experiencias, que a pesar de tener muy distintas edades, personalidades y de no conocernos, la experiencia por sí misma nos unió y permitió acercarnos, conocernos y valorarnos como personas.

\section{A manera de cierre}

La movilidad internacional que vivieron 31 estudiantes de la Maestría en Educación fue breve, de apenas una semana, a pesar de lo cual los participantes señalan haber obtenido aprendizajes cuantiosos y de diversa naturaleza. Esto incluye cuestiones académicas y profesionales, oportunidades de crecimiento personal y experiencias de vida. La experiencia no 
fue igual para todos, cada estudiante destacó en su trabajo integrador aquello que le resultó relevante y llegó a ser opuesto a lo identificado por otro compañero. Hay quienes incluso reforzaron estereotipos, lo cual no era una meta de la experiencia. A pesar de esto último, lo más relevante de la movilidad internacional es que hubo aprendizajes identificados por todos los participantes, algunos que se podrían transferir, otros que los nutren intelectual o personalmente. Por todo esto, las experiencias de movilidad estudiantil internacional son recomendables, tanto para los estudiantes como para las instituciones de educación superior.

\section{REFERENCIAS}

ALCÓN, E. La internacionalización de los estudiantes universitarios. La cuestión Universitaria, n. 7, p. 32-39, 2011.

ANUIES. Movilidad estudiantil. [21--]. Disponible en: http://www.anuies.mx/programas-yproyectos/cooperacion-academica-nacional-e-internacional/cooperacion-academicainternacional/movilidad-estudiantil. Acceso en: 10 sep. 2020.

BEELEN, J.; JONES, E. Redefining internationalization at home. In: CURAJ, A.; MATEI, L.; PRICOPIE, R.; SALMI, J.; SCOTT, P. (Eds.). The European higher education area. Between critical reflections and future policies. Springer, Cham, 2015. p. 59-72. DOI: https://link.springer.com/chapter/10.1007\%2F978-3-319-20877-0_5

CHANTRAIN, H. Mobility of students, academic and administrative staff: A basis for establishing a European Higher Education area. Folia Phoniatrica et Logopaedica, v. 62, n. 5, p. 234-237. DOI: https://doi.org/10.1159/000314786

DE WIT, H. Globalización e internacionalización de la educación superior RUSC. Universities and Knowledge Society Journal, v. 8, n. 2, p. 77-84. Disponible en: https://www.redalyc.org/articulo.oa?id=78018793007. Acceso en: 10 sep. 2020.

FRANCO-LEAL, N.; SOETANTO, D.; CAMELO-ORDAZ, C. Do they matter? The role of non-academics in the internationalization of academic spin-offs. Journal of International Entrepreneurship, v. 14, n. 3, p. 410-440, 2016. DOI: https://doi.org/10.1007/s10843-0160184-X

GALLEGO, J. L.; SALVADOR, F. Contenidos y competencias básicas en el proceso didáctico. In: MEDINA, A.; SALVADOR, F. (Eds.). Didáctica general. 2. ed. Madrid, España: Pearson Prentice Hall y UNED, 2009. p. 139-166.

GÁRATE, A.; ROCHA, J. (Eds.). Plan de desarrollo CETYS 2020. 2011. Disponible em: http://www.cetys.mx/wp-content/uploads/2017/02/PlanFINAL.pdf. Acceso en: 10 sep. 2020.

GARCÍA, J. J. Movilidad estudiantil internacional y cooperación educativa en el nivel superior de educación. Revista Iberoamericana de Educación, v. 61, p. 59-76, 2013. DOI: https://doi.org/10.35362/rie610600 
GREEN, W.; MERTOVA, P. Transformalists and transactionists: towards a more comprehensive understanding of academics' engagement with "internationalisation of the curriculum". Research in Comparative and International Education, v. 11, n. 3, p. 229246, 2016. DOI: https://doi.org/10.1177/1745499916662372

KNIGHT, J. Updating the definition of internationalization. International Higher Education, v. 33, n. 6, p. 2-3, 2015. DOI: https://doi.org/10.6017/ihe.2003.33.7391

LANDINELLI, J. El sentido de la internacionalización universitaria en los procesos de integración regional. Boletin IESALC Informa de Educación Superior, n. 211, 2010. Disponible en:

http://www.unesco.org.ve/index.php?option=com_content\&view=article\&id=2421\%3Aelsentido-de-la-internacionalizacion-universitaria-en-los-procesos-de-integracionregional\&catid=126\%3Anoticias-pagina-nueva\&Itemid=712\&lang=es. Acceso em: 10 sep. 2020.

Leask, B. Internationalizing the curriculum. Estados Unidos: Routledge, 2015.

MAYRING, P. Qualitative content analysis: theoretical foundation, basic procedures and software solution. Klagenfurt, Austria: Social Science Open Access Repository. Disponible en: http://nbn-resolving.de/urn:nbn:de:0168-ssoar-395173. Acceso em: 10 sep. 2020.

SALDAÑA, J. The coding manual for qualitative researchers. 3. ed. Londres, Inglaterra: SAGE, 2016.

\section{Cómo referenciar este artículo}

BONILLA ESQUIVEL, J. L.; MONTES SILVA, M. E. Cursos breves en el extranjero: aprendizajes logrados por estudiantes de posgrado en educación. Revista Ibero-Americana de Estudos em Educação, Araraquara, v. 15, n. esp. 4, p. 2577-2586, dez., 2020. e-ISSN: 19825587. DOI: https://doi.org/10.21723/riaee.v15iesp4.14505

Remitido el: 10/09/2019

Revisiones requeridas el: 10/01/2020

Aprobado el: $30 / 04 / 2020$

Publicado el: 01/12/2020 